\title{
Copy number variation of ribosomal DNA and Pokey transposons in natural populations of Daphnia
}

\author{
Shannon HC Eagle and Teresa J Crease
}

\begin{abstract}
Background: Despite their ubiquity and high diversity in eukaryotic genomes, DNA transposons are rarely encountered in ribosomal DNA (rDNA). In contrast, R-elements, a diverse group of non-LTR retrotransposons, specifically target rDNA. Pokey is a DNA transposon that targets a specific rDNA site, but also occurs in many other genomic locations, unlike R-elements. However, unlike most DNA transposons, Pokey has been a stable component of Daphnia genomes for over 100 million years. Here we use GPCR to estimate the number of $18 \mathrm{~S}$ and $28 \mathrm{~S}$ ribosomal RNA genes and Pokey elements in rDNA (rPokey), as well as other genomic locations (gPokey) in two species of Daphnia. Our goals are to estimate the correlation between (1) the number of $18 \mathrm{~S}$ and $28 \mathrm{~S}$ rRNA genes, (2) the number of 285 genes and rPokey, and (3) the number of rPokey and gPokey. In addition, we ask whether Pokey number and distribution in both genomic compartments are affected by differences in life history between D. pulex and D. pulicaria.

Results: We found differences in $18 \mathrm{~S}$ and $28 \mathrm{~S}$ gene number within isolates that are too large to be explained by experimental variation. In general, Pokey number within isolates is modest $(<20)$, and most are gPokey. There is no correlation between the number of rRNA genes and rPokey, or between rPokey and gPokey. However, we identified three isolates with unusually high numbers of both rPokey and gPokey, which we infer is a consequence of recent transposition. We also detected other rDNA insertions (rlnserts) that could be degraded Pokey elements, Relements or the divergent PokeyB lineage recently detected in the Daphnia genome sequence. Unlike rPokey, r/nserts are positively correlated with rRNA genes, suggesting that they are amplified by the same mechanisms that amplify rDNA units even though rPokey is not. Overall, Pokey frequency and distribution are similar in D. pulex and D. pulicaria suggesting that differences in life history have no impact on Pokey.

Conclusions: The possibility that many rDNA units do not contain a copy of both $18 \mathrm{~S}$ and $28 \mathrm{~S}$ genes suggests that rDNA is much more complicated than once thought, and warrants further study. In addition, the lack of correlation between rPokey, gPokey and rDNA unit numbers suggests that Pokey transposition rate is generally very low, and that recombination, in combination with natural selection, eliminates rPokey much faster than gPokey. Our results suggest that further research to determine the mechanisms by which Pokey has escaped complete inactivation by its host (the usual fate of DNA transposons), would provide important insights into transposon biology.
\end{abstract}

Keywords: Daphnia, transposons, Pokey, ribosomal RNA genes, copy number variation, quantitative PCR

\footnotetext{
* Correspondence: tcrease@uoguelph.ca

Department of Integrative Biology, University of Guelph, Guelph, ON N1G

2W1, Canada
} 


\section{Background}

Transposable elements (TEs) are segments of DNA that can move or transpose around the genome [1]. Despite the fact that some have been co-opted for cellular functions by their host [2], TEs are generally considered to be detrimental because they can disrupt function when they insert into or near genes, or promote ectopic recombination, which can lead to chromosome rearrangements [3]. Moreover, their transposition may have energy costs [3] and the epigenetic mechanisms used by the host to control their expression can also alter gene function [4]. Even so, transposons are ubiquitous in eukaryotes and have become basic genomic components like exons, introns, telomeres and centromeres $[5,6]$.

Ribosomal (r)DNA is a multigene family composed of repeated units each containing an $18 \mathrm{~S}, 5.8 \mathrm{~S}$ and $28 \mathrm{~S}$ rRNA gene, and spacers (Figure 1). Due to the highly repetitive nature of rDNA, it usually evolves in a concerted fashion such that rDNA units are more similar within than between species [7]. The primary mechanisms responsible for this phenomenon are thought to be unequal crossing over and gene conversion [7]. Given the constant turnover of rDNA units caused by this recombination, and the strong purifying selection that operates on the rRNA genes, it is somewhat surprising that some TEs insert specifically into rDNA [7]. On the other hand, it has been argued that its repetitive nature makes rDNA an ideal niche for TEs. For example, recombination can increase the number of rDNA units and thus provide new insertion sites, or it can remove inactive TEs to reopen sites into which active elements can insert. Moreover, rRNA genes are highly transcribed providing many opportunities for expression of TE-encoded genes [8]. TEs that specifically target rDNA typically insert into

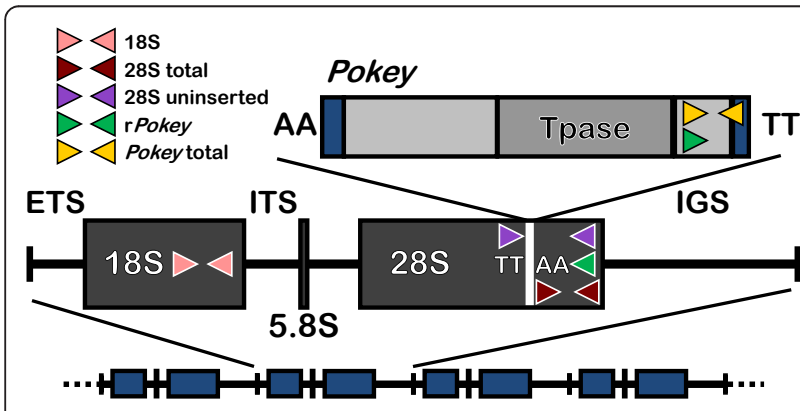

Figure 1 Eukaryotic rDNA structure and location of qPCR primers to estimate Daphnia rDNA and Pokey number. Primers are indicated by short arrows. Pokey inserts into a specific TTAA site in the $28 \mathrm{~S}$ gene, which is duplicated at either end of the inserted element. 18S, $18 \mathrm{~S}$ rRNA coding region; 28S, $28 \mathrm{~S}$ rRNA coding region; 5.8S, 5.8S rRNA coding region; IGS, intergenic spacer; ETS, external transcribed spacer; ITS, internal transcribed spacer; Tpase, transposase gene. The darker vertical bars at either end of Pokey represent the Terminal Inverted Repeats. either the $18 \mathrm{~S}$ or the $28 \mathrm{~S}$ genes, which make the genes non-functional $[9,10]$. However, the number of rDNA units usually exceeds the minimum required for host viability, and thus insertion of TEs into some rDNA units may have little impact on host fitness $[8,11]$. Indeed, some species are able to recognize and preferentially deactivate rDNA units containing insertions [12]. The most extensively studied rDNA-specific TEs are the Class I non-LTR retrotransposons, R1 and R2 [11], which are common in arthropods. R2 and other related lineages have also been found in diverse animal phyla, including Chordata, Echinodermata, Plathyhelminthes, Rotifera and Cnidaria $[9,10,13]$. These TEs typically undergo long periods of stable, vertical inheritance and diverge congruently with their hosts [11].

Pokey is a Class II DNA transposon in the piggyBac superfamily that ranges in size from 4 to $10 \mathrm{~kb}$ and contains the terminal inverted repeats (TIRs) characteristic of this element class [14,15]. Many copies of Pokey encode a transposase whose ability to excise the element has been confirmed in a yeast excision assay [15]. Pokey was first identified in the rDNA of the cladoceran crustacean, Daphnia pulex [16] and is widespread in the subgenus Daphnia $[17,18]$. The only other taxa in which Pokey elements have been discovered are the silk moth, Bombyx mori [19] and the tunicate, Ciona savignyi [20]. Pokey is unique among DNA transposons in that it targets a specific TTAA site in the $28 \mathrm{~S}$ genes of Daphnia (Figure 1) where it has undergone stable, vertical inheritance for millions of years [17]. Moreover, this TTAA site is only 4 nucleotides (nt) from the insertion site of $\mathrm{R} 2$, and 64 nt from the insertion site of R1. However, unlike R-elements, which tend to specialize on rDNA (but see [9]), Pokey also inserts into many other locations throughout the Daphnia nuclear genome [21-23], although only a single copy has been reported from the nuclear genomes of B. mori and C. savignyi.

Daphnia are freshwater crustaceans (Cladocera, Crustacea) that inhabit standing water from shallow, ephemeral puddles to deep stratified lakes. They typically reproduce by cyclic parthenogenesis in which production of direct-developing embryos by apomictic parthenogenesis alternates with the meiotic production of ephippial (diapausing) eggs that require fertilization. Males are produced apomictically and sex-determination is environmental. Populations that inhabit temporary bodies of water that either dry up or freeze during the year are re-established from ephippial offspring annually. In contrast, adults can persist year-round in permanent ponds and lakes, and recruitment of individuals from ephippial eggs may be sporadic. In addition, some lineages have completely lost the capacity for sexual reproduction and produce their ephippial eggs apomictically (obligate parthenogenesis). 
Species in the Daphnia pulex complex typically inhabit ponds and small unstratified lakes that lack fish. However, one species in this complex, Daphnia pulicaria, has invaded large stratified lakes in North America [24], and is able to tolerate predation by fish by taking refuge in the cold hypolimnion during the day [25]. The transition to lake habitats has led to substantial changes in physiology and life history [26,27]. Even so, $D$. pulicaria produces viable hybrids with other members of the $D$. pulex species complex. Hybrids typically occur in ponds or disturbed, intermediate habitats, and reproduce by obligate parthenogenesis [28]. Despite this hybridization, lake populations of $D$. pulicaria remain genetically [29] and ecologically [26,27] distinct from the other species in the complex.

The occurrence of Pokey in both rDNA (rPokey) and other genomic locations (gPokey) in Daphnia provides a unique opportunity to study its distribution in different genomic compartments and under different modes of reproduction (with and without meiosis). For example, using the PCR-based technique TE Display [30], Valizadeh and Crease [21] and Schaack et al. [23] compared the relative load of gPokey in cyclically and obligately parthenogenetic populations of $D$. pulex in North America. The results of both studies show that cyclical individuals carry, on average, more elements than obligate individuals, which is consistent with predictions about the effect of breeding system on TE dynamics [31]. However, it is not possible to estimate rPokey or rRNA gene number with TE Display, so the dynamics of $\mathrm{rPo}$ key have not yet been determined. On the other hand, Averbeck and Eickbush [32] measured R1 and R2 number in the rDNA of replicate laboratory lines of Drosophila melanogaster and found that R2 number remained relatively constant through time, but changes in R1 number were strongly correlated with changes in the overall size of the rDNA locus, which varied from 140 to 310 units after 400 generations of laboratory culture. Moreover, transposition of full-length elements accounted for most of the change in R1 number. This level of rRNA gene variation was also observed in replicate, clonally-propagated lines of Daphnia obtusa, in which the haploid number of $18 \mathrm{~S}$ genes varied from 53 to 233 after only 90 generations [33].

In this study, we use quantitative PCR (qPCR) to measure the number of $18 \mathrm{~S}$ and $28 \mathrm{~S}$ rRNA genes, as well as rPokey and gPokey in 16 pond populations (43 isolates) of $D$. pulex and 6 lake populations (26 isolates) of $D$. pulicaria (Figure 2, Additional file 1). Our objectives are to estimate the correlation between (1) the number of $18 \mathrm{~S}$ and $28 \mathrm{~S}$ genes, (2) the number of $28 \mathrm{~S}$ genes and rPokey, and (3) the number of rPokey and gPokey. In addition, we ask if life history differences between $D$. pulex and D. pulicaria impact Pokey frequency and

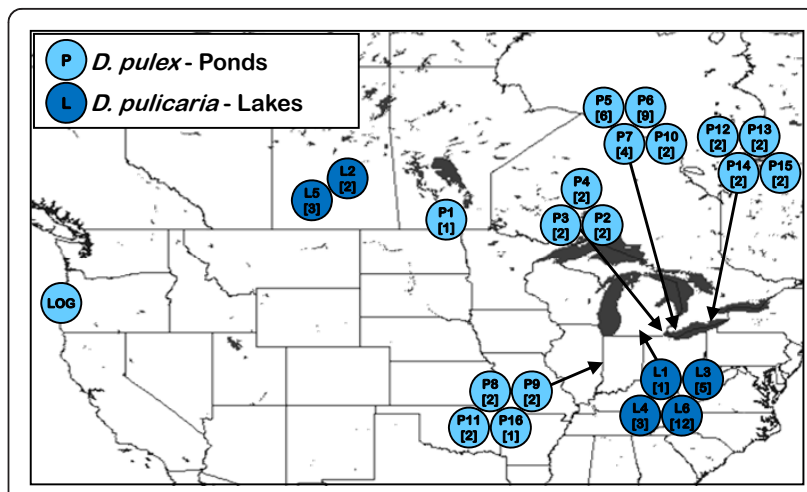

Figure 2 Location of $D$. pulex and $D$. pulicaria populations sampled for this study. Daphnia pulex were sampled from ponds (P) and D. pulicaria from lakes (L). Each circle contains the population code and the number of isolates analyzed in brackets.

distribution. We expect a positive correlation between $18 \mathrm{~S}$ and $28 \mathrm{~S}$ gene number if most rDNA units are complete, although this has rarely been tested. Moreover, if rates of transposition and/or elimination by the host vary in response to the number of available insertion sites, we expect to observe a positive association between rPokey and $28 \mathrm{~S}$ gene number. We also expect a positive correlation between rPokey and gPokey number if rates of transposition and/or rates of element elimination are similar both inside and outside of rDNA. Finally, because rates of recruitment of sexually-produced offspring differ between pond populations, which are re-established each year from ephippial hatchlings, and lake populations, which often undergo extended periods of clonal reproduction, there may be more opportunities for transposition in pond populations and thus higher Pokey load.

\section{Results}

We calculated the haploid number of rRNA genes (18S and 28S) and Pokey relative to two single-copy reference genes, Tif (a transcription initiation factor) and Gtp (a member of the RAB subfamily of small GTPases) [33]. The number of $28 \mathrm{~S}$ was estimated in two regions: downstream of the Pokey insertion site (total 28S or t28S) and across the Pokey insertion site (uninserted 28S or u28S, Figure 1), which allowed us to determine if there are insertions present in that position that would not amplify with our Pokey primers (for example, R2). In addition, we estimated the number of rPokey and total Pokey elements in the genome (Figure 1, Additional file 2).

We expect the number of one reference gene relative to the other to be close to 1 , and this is often the case (Table 1, Additional file 2). However, values of Tif relative to Gtp (TG ratio) vary from 0.65 to 1.32 across the 69 Daphnia isolates, with a mean of 0.90 for D. pulex and 0.92 for $D$. pulicaria (Table 1). One explanation for 
Table 1 Pokey and rRNA gene number in Daphnia from North America

\begin{tabular}{|c|c|c|c|c|c|c|c|c|c|c|}
\hline \multirow[b]{2}{*}{ Species } & \multirow[b]{2}{*}{$\begin{array}{l}\text { Number of } \\
\text { populations }\end{array}$} & \multirow[b]{2}{*}{$\begin{array}{l}\text { Number of } \\
\text { isolates }\end{array}$} & \multicolumn{8}{|c|}{ Number of genes mean/range } \\
\hline & & & Tif:Gtp ${ }^{1}$ & $18 S$ & $\mathrm{t} 28 \mathrm{~S}^{2}$ & rPokey ${ }^{3}$ & gPokey ${ }^{4}$ & rlnserts $^{5}$ & $\begin{array}{l}\text { \% Pokey in } \\
285^{6}\end{array}$ & $\begin{array}{c}\% \\
\text { rlnserts }^{7}\end{array}$ \\
\hline D. pulex & 16 & 43 & $\begin{array}{c}0.90 \\
0.65 \text { to } \\
1.31\end{array}$ & $\begin{array}{l}221.0 \\
94 \text { to } \\
489.5\end{array}$ & $\begin{array}{l}260.0 \\
88 \text { to } \\
724.5\end{array}$ & $\begin{array}{c}2.1 \\
0 \text { to } 12\end{array}$ & $\begin{array}{l}9.6 \\
4 \text { to } 18\end{array}$ & $\begin{array}{c}21.1 \\
0 \text { to } \\
113.5\end{array}$ & $\begin{array}{c}17.2 \\
0 \text { to } 63.2\end{array}$ & $\begin{array}{c}6.5 \\
0 \text { to } 28.6\end{array}$ \\
\hline D. pulicaria & 6 & 26 & $\begin{array}{c}0.92 \\
0.70 \text { to } \\
1.32\end{array}$ & $\begin{array}{c}215.1 \\
97 \text { to } \\
444\end{array}$ & $\begin{array}{c}266.1 \\
109 \text { to } \\
654.5\end{array}$ & $\begin{array}{l}6.6 \\
0 \text { to } \\
44.5\end{array}$ & $\begin{array}{c}9.5 \\
4.5 \text { to } \\
24\end{array}$ & $\begin{array}{c}14.9 \\
0 \text { to } 76\end{array}$ & $\begin{array}{c}27.1 \\
0 \text { to } 79.2\end{array}$ & $\begin{array}{c}4.6 \\
0 \text { to } 19.7\end{array}$ \\
\hline $\begin{array}{c}\text { D. pulicaria - } \\
3^{8}\end{array}$ & 6 & 23 & $\begin{array}{c}0.93 \\
0.70 \text { to } \\
1.32\end{array}$ & $\begin{array}{c}217.3 \\
97 \text { to } \\
444\end{array}$ & $\begin{array}{c}273.9 \\
109 \text { to } \\
654.5\end{array}$ & $\begin{array}{c}2.7 \\
0 \text { to } 7.5\end{array}$ & $\begin{array}{c}8.9 \\
4.5 \text { to } \\
18\end{array}$ & $\begin{array}{c}16.9 \\
0 \text { to } 76\end{array}$ & $\begin{array}{c}21.2 \\
0 \text { to } 57.7\end{array}$ & $\begin{array}{c}5.2 \\
0 \text { to } 19.7\end{array}$ \\
\hline
\end{tabular}

1. Number of Tif genes relative to Gtp genes.

2. Denotes total $28 \mathrm{~S}$ genes.

3. Denotes Pokey in 285 genes.

4. Denotes Pokey in other genome sites. Calculated as (total Pokey - rPokey).

5. Denotes other rDNA inserts, calculated as (t28S-u28S-rPokey) where u28S is uninserted $28 \mathrm{~S}$ genes.

6. Calculated as (rPokey/total Pokey x 100).

7. Calculated as (r/nserts/t28S $\times 100)$.

8. Results obtained after removing three $D$. pulicaria isolates with high Pokey load (see text)

the extreme values is that there are three copies of one gene instead of the two expected, in which case we expect a ratio of 0.67 (3 Gtp) or 1.5 (3 Tif). Further analysis (Additional file 3) suggests that there are three copies of Gtp in one isolate (TG ratio $=0.65)$ and three copies of Tif in the four isolates (TG ratios $\geq 1.25$ ). Estimates of gene number based on the duplicated gene were adjusted by multiplying them by 1.5 in these isolates.

\section{Variation in rRNA gene number}

The haploid number of $18 \mathrm{~S}$ varies from 94 to 489.5 across all 69 Daphnia isolates (Table 1), and the mean value is not significantly different between the two species $(t=-0.26, d f=50, P=0.79)$. An even wider range of variation was observed for $28 \mathrm{~S}$ (88 to 724.5), but again, the mean value does not differ significantly between species $(t=0.18, d f=48, P=0.86)$. Similarly, there is no significant difference between the mean number of $18 \mathrm{~S}$ and $28 \mathrm{~S}$ within each species (D. pulicaria $\mathrm{t}=-1.52, \mathrm{df}=43, \mathrm{P}=0.14 ; D$. pulex $\mathrm{t}=-1.64, \mathrm{df}$ $=74, \mathrm{P}=0.10$ ).

The estimates of $18 \mathrm{~S}$ and $28 \mathrm{~S}$ number within each isolate are significantly correlated (Table 2, Figure 3), but the slope of the line generated by plotting them relative to one another is 1.26 , which is substantially higher than the expected value of 1 . Moreover, $18 \mathrm{~S}$ and $28 \mathrm{~S}$ numbers are significantly different in $61 \%$ of the 69 isolates with $28 \mathrm{~S}$ exceeding $18 \mathrm{~S}$ in $76 \%$ of these cases (Additional files 2 and 4).

Table 2 Correlations between Pokey and rRNA gene number in Daphnia from North America

\begin{tabular}{|c|c|c|c|c|c|c|c|}
\hline Species & X-axis & Y-axis & slope & y-intercept & $\mathrm{R}^{2}$ & $P$-value & Figure \\
\hline All isolates & 185 & 285 & 1.257 & -12.69 & 0.690 & 0.000 & 3 \\
\hline \multirow[t]{4}{*}{ D. pulex } & $28 S$ & $428 S^{1}$ & 0.850 & 15.71 & 0.976 & 0.000 & $5 a$ \\
\hline & $28 S$ & rlnserts ${ }^{2}$ & 0.150 & -17.84 & 0.556 & 0.000 & $5 a$ \\
\hline & $28 S$ & rPokey ${ }^{3}$ & -0.00002 & 2.14 & 0.000 & 0.995 & $5 a$ \\
\hline & gPokey ${ }^{4}$ & rPokey & -0.016 & 2.29 & 0.0005 & 0.884 & $4 b$ \\
\hline \multirow[t]{4}{*}{ D. pulicaria } & 285 & u28S & 0.907 & 3.26 & 0.981 & 0.000 & $5 b$ \\
\hline & $28 \mathrm{~S}$ & rlnserts & 0.104 & -12.80 & 0.475 & 0.0001 & $5 b$ \\
\hline & $28 S$ & rPokey & -0.011 & 9.54 & 0.020 & 0.493 & $5 b$ \\
\hline & gPokey & rPokey & 1.475 & -7.48 & 0.290 & 0.005 & $4 b$ \\
\hline D. pulicaria- $3^{5}$ & gPokey & rPokey & 0.123 & 1.62 & 0.031 & 0.420 & $4 b$ \\
\hline
\end{tabular}

1. Denotes uninserted 28S rRNA genes.

2. Denotes other rDNA inserts calculated as (t28S-u28S-rPokey) where t28S is total $28 \mathrm{~S}$ genes.

3. Denotes Pokey in 285 genes.

4. Denotes Pokey in other genome sites. Calculated as (total Pokey - rPokey).

5. Results obtained after removing three $D$. pulicaria isolates with high Pokey load (see text). 


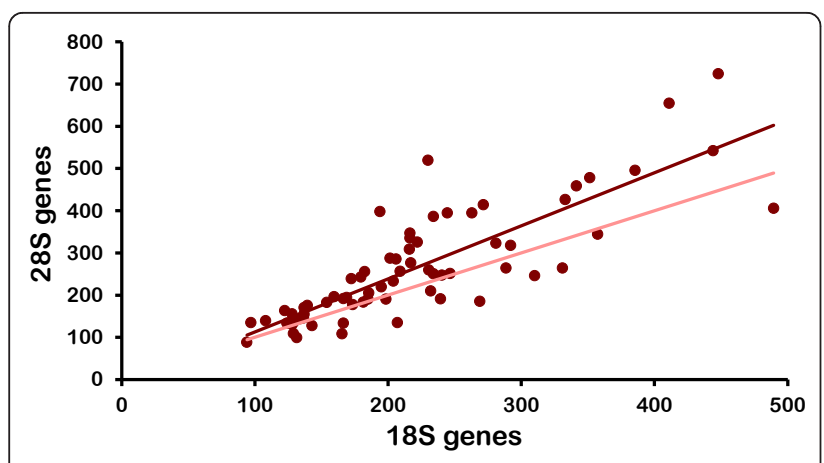

Figure 3 Correlation between $18 \mathrm{~S}$ and 28S rRNA genes in $D$. pulex and $D$. pulicaria. The haploid number of each gene is plotted. The lighter line was generated by plotting the number of 185 genes on both axes.
Variation in Pokey number

Pokey number in 28S (rPokey) varies from 0 to 44.5 , with a mean of 2.1 for $D$. pulex and 6.6 for $D$. pulicaria (Table 1, Figure 4a), but these differences are not significant $(\mathrm{t}=1.96, \mathrm{df}=26, \mathrm{P}=0.06)$. The higher mean for D. pulicaria is due to three isolates with $23.5,40$ and 44.5 rPokey (Figure 4a, Additional file 2). If we exclude these isolates, the mean decreases to 2.7 (Table 1). rPokey number is 7.5 or fewer in all other $D$. pulicaria isolates, and three have none. Similarly, we did not detect rPokey in three D. pulex isolates. Moreover, the haploid rPokey number is only 0.5 in a total of eight isolates (Additional file 2), which means they have a single copy among all of their $28 \mathrm{~S}$ genes. We tested these results in 26 isolates using end-point PCR, and they correspond

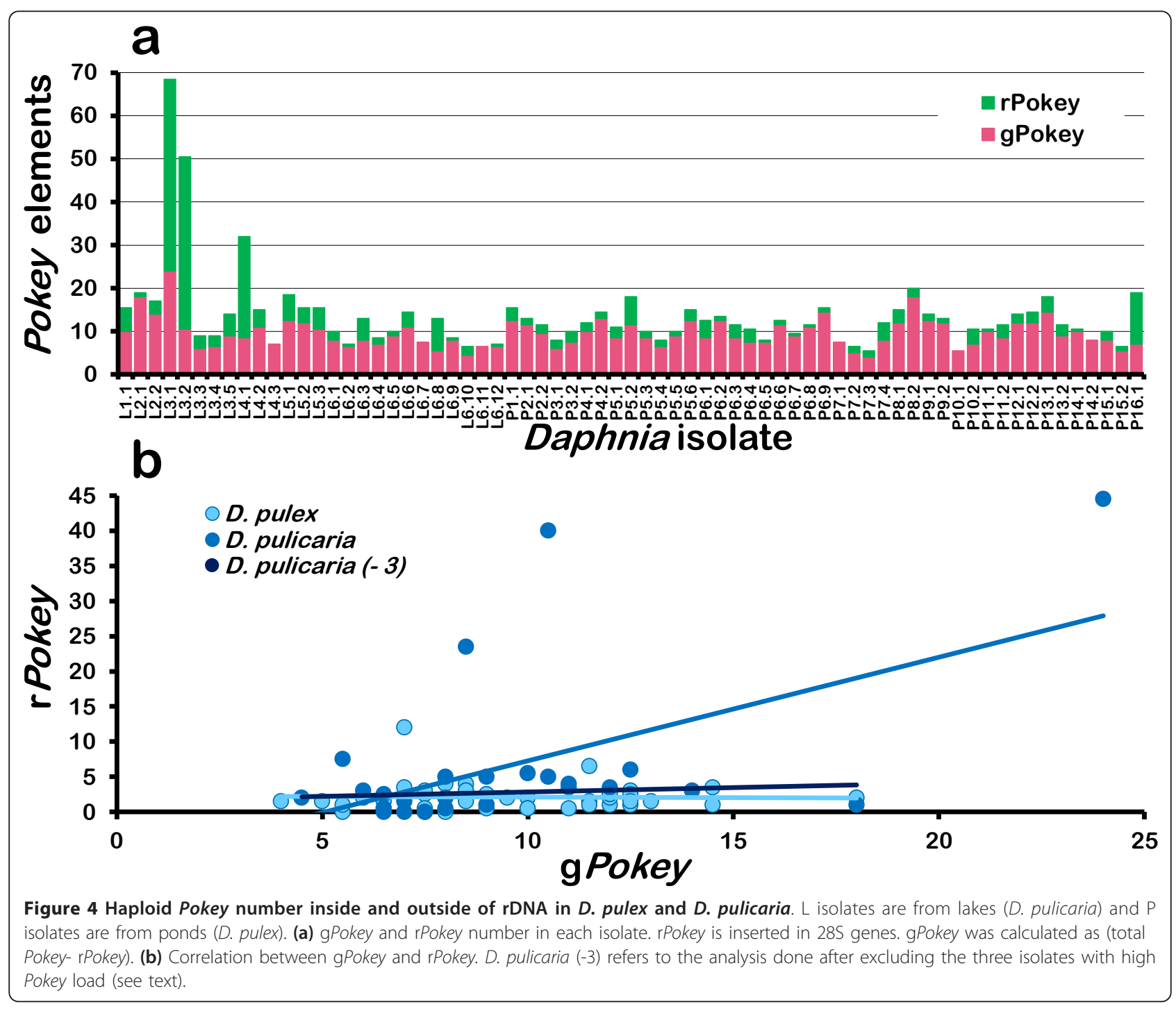


with six exceptions in which we were not able to amplify rPokey from isolates that have it based on qPCR. The qPCR estimate of rPokey is 0.5 in three of these isolates, 1 in one isolate and 2 in two isolates.

Pokey number outside of rDNA (gPokey) ranges from 4 to 24 with a mean of 9.6 in $D$. pulex and 9.5 in $D$. pulicaria (Table 1, Figure 4a), and this difference is not significant $(\mathrm{t}=-0.09, \mathrm{df}=40, \mathrm{P}=0.93)$. The same three $D$. pulicaria isolates with high rPokey number also have high gPokey number $(8.5,10.5,24)$. If we exclude these isolates, mean gPokey decreases to 8.9, which is lower than that in $D$. pulex (9.6) but not significantly different. Overall, gPokey is present in all isolates, and, with the exception of the three unusual $D$. pulicaria isolates, it is much more numerous than rPokey (Table 1, Figure 4a). On average, less than $28 \%$ of the Pokey elements in an isolate are located in $28 \mathrm{~S}$ genes (Table 1).

There is no correlation between rPokey and gPokey number in $D$. pulex, but they are highly correlated in $D$. pulicaria (Table 2, Figure 4b). This correlation is mainly driven by the three unusual isolates with high Pokey load. We recalculated the correlation after excluding these isolates, and it is not significant.

\section{Variation in $28 \mathrm{~S}$ gene insertion number}

In general, the total number of $28 \mathrm{~S}$ genes (t28S) exceeds the number of uninserted $28 \mathrm{~S}$ genes (u28S), as expected. However, u28S is higher than t $28 \mathrm{~S}$ in 20 isolates. In addition, the sum of u28S plus rPokey exceeds $\mathrm{t} 28 \mathrm{~S}$ in four isolates (Additional file 2). In these cases, we used the qPCR estimate of $\mathrm{t} 28 \mathrm{~S}$ and calculated u28S as (t28SrPokey).

Differences in number between t28S and u28S (Table 1, Additional files 2 and 4) suggest that some genes contain insertions other than Pokey, or their Pokey elements do not bind to our qPCR primers. We refer to these as other rDNA inserts (rInserts). rInsert number ranges from 0 to 113.5 in D. pulex $($ mean $=21.1)$ and from 0 to 76 in D. pulicaria (mean $=14.9$, Table 1 ), and the means are not significantly different $(t=-1.06, \mathrm{df}=60$, $P=0.29)$. On average, rinserts occur in less than $10 \%$ of $28 \mathrm{~S}$ (Table 1). It is likely that some of the difference between $\mathrm{t} 28 \mathrm{~S}$ and $\mathrm{u} 28 \mathrm{~S}$ is due to experimental variation, and indeed this likely explains most cases where u28S exceeds t28S (as described above). However, experimental variation is not likely to be the only explanation because the slope of the line generated by plotting u28S relative to $\mathrm{t} 28 \mathrm{~S}$ is significantly lower than the expected value of 1 in both species, but the correlation between t28S and u28S is $>97 \%$ (Table 2, Figure 5).

There is a strong positive correlation (approximately $50 \%$ ) between $\mathrm{t} 28 \mathrm{~S}$ and rInserts in both species (Table 2 ), although rInsert number increases at a substantially slower rate $(D$. pulex slope $=0.15, D$. pulicaria slope $=$

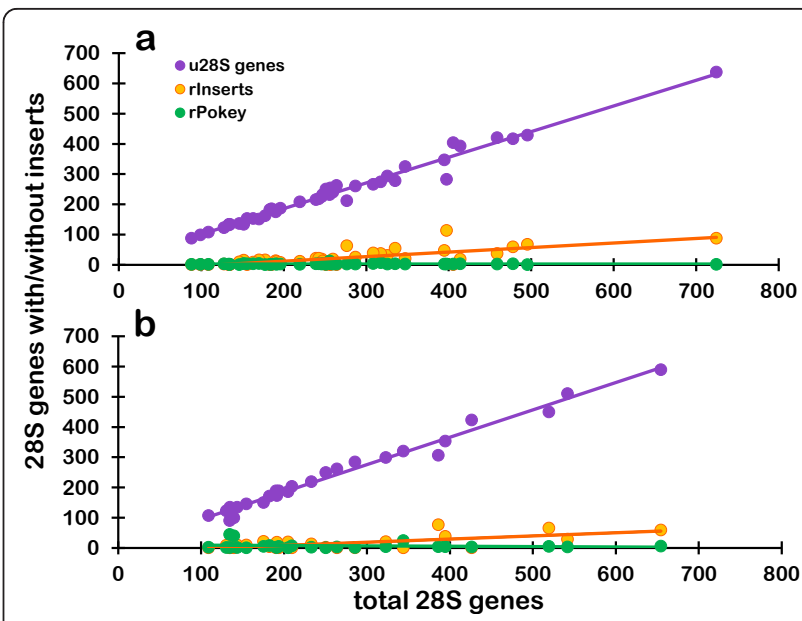

Figure 5 Correlation between total $28 \mathrm{~S}$ genes and genes with and without insertions. (a) Daphnia pulex. (b) Daphnia pulicaria. u28S genes denotes uninserted 285 genes. rPokey is inserted in 285 genes. The number of other rDNA insertions (r/nserts) was calculated as (t28S-u28S-rPokey) where $\mathrm{t}$ is total $28 \mathrm{~S}$ genes.

0.10, Figure 5) than does u28S. Moreover, these results are very different from the situation with $\mathrm{rPokey}$, which shows no correlation with t28S (Table 2, Figure 5) and is present in very low numbers in all but three $D$. pulicaria isolates (Figure 4).

\section{Comparison of rRNA gene and Pokey number based on qPCR and genome sequencing}

Colbourne et al. [34] estimated the haploid number of rDNA units from the $D$. pulex genome sequence by comparing the depth of sequence coverage in the trace files across the entire rDNA repeat and the average single-copy gene. The estimate they reported is 435 . In addition, Schaack et al. [22] searched the annotated genome sequence (which does not include rDNA) for Pokey using BLASTN and RepeatMasker. Their estimates

Table 3 Pokey and rRNA gene number in two clonal lines of Daphnia pulex

\begin{tabular}{|c|c|c|c|c|c|c|c|}
\hline \multirow[b]{2}{*}{ Isolate $^{1}$} & \multicolumn{7}{|c|}{ Gene Region } \\
\hline & $18 S$ & $t 28 S^{2}$ & $\mathrm{u} 28 \mathrm{~S}^{3}$ & rPokey 4 & gPokey 5 & rlnserts $^{6}$ & $\begin{array}{l}\text { Tif: } \\
\text { Gtp }\end{array}$ \\
\hline $\begin{array}{c}\log 50- \\
11\end{array}$ & 301 & 540.5 & 445 & 2.5 & 37 & 93 & 0.84 \\
\hline $\begin{array}{c}\log 50- \\
12\end{array}$ & 245.5 & 362.5 & 349 & 4 & 25.5 & 9.5 & 0.88 \\
\hline
\end{tabular}

1. Log50 is the isolate from which the $D$. pulex genome sequence was generated [34]. Log50-11 and Log50-12 are clonally-propagated lines derived from Log50 [22].

2. Denotes total $28 \mathrm{~S}$ genes.

3. Denotes uninserted $28 \mathrm{~S}$ genes.

4. Denotes Pokey in $28 \mathrm{~S}$ genes.

5. Denotes Pokey in other genome sites. Calculated as (total Pokey - rPokey).

6. Denotes other rDNA inserts, calculated as (t28S-u28S-rPokey).

7. Number of Tif genes relative to Gtp genes 
based on these two approaches are 35 and 123, respectively.

In 2004, Schaack et al. [22] started a large number of clonally-propagated lines of the $\log 50 \mathrm{D}$. pulex isolate (whose genome was sequenced) from the lab culture that was established from the original female, collected in 2000. We obtained DNA extracted from two of these lines (Log50-11 and Log50-12) in 2006, approximately 40 generations after they were initiated, and estimated their Pokey and rRNA gene number using qPCR to compare them with estimates based on the genome sequence. The number of $18 \mathrm{~S}$ is lower than $28 \mathrm{~S}$ in both isolates (Table 3) with a ratio of 1:1.8 for Log50-11 and $1: 1.5$ for Log50-12. The mean number of $18 \mathrm{~S}$ and $28 \mathrm{~S}$ is 304 (Log50-12) and 421 (Log50-11), which is well within the range of variation observed within clonal $D$. obtusa lines over 90 generations [33]. Moreover, the Log50-11 estimate is very close to the value generated from the genome sequence (435).

Our estimates of gPokey number (37 and 25.5, Table 3) are also very similar to the estimate (35) obtained from the BLASTN search of the genome sequence. However, all three estimates are substantially lower than the one obtained from the genome sequence using RepeatMasker (123), suggesting that the similarity criteria used for the BLASTN search identified similar elements to the ones we detected with qPCR. Overall, our qPCR estimates are very similar to those obtained from the genome sequence.

\section{Discussion}

\section{Variation in rRNA gene number}

Variation in physiological responses to environmental heterogeneity has been linked with variation in rDNA copy number in diverse organisms (reviewed in [35]). Moreover, Paredes et al. [36] recently showed that differences in rDNA copy number have a significant effect on the level of expression of thousands of other genes in the Drosophila genome, which further reinforces the idea that rDNA copy number variation may be an important source of phenotypic and regulatory variation in natural populations. This is especially relevant for Daphnia, which is becoming an important model system for the study of genetic and physiological responses to environmental variation since publication of the genome sequence and development of other genomics tools [34].

In this study, we detected substantial variation in rRNA gene number within and between natural populations of D. pulex and D. pulicaria. The mean (approximately 220 for $18 \mathrm{~S}$, approximately 245 for $28 \mathrm{~S}$ ) and range is similar in both species with no values $<80$ observed. However, the highest values are up to eight times larger than the smallest, which means that the diploid number of rRNA genes can vary by more than an order of magnitude among individuals of the same species. Such a large range of variation has been observed in natural populations of a diverse array of organisms, including plants, arthropods and vertebrates [35] and thus, Daphnia are not unusual in this regard.

Using computer simulations, Zhang et al. [8] showed that rDNA locus size is primarily a function of the rate of sister chromatid exchange, which creates high levels of variation among individuals and large rDNA loci. In contrast, high rates of interchromosomal exchange tend to reduce variation among individuals, and reduce the overall size of the rDNA locus. This model suggests that the highly variable number of rRNA genes in $D$. pulex and $D$. pulicaria results from higher rates of intrachromosomal than interchromosomal exchange, and this is indeed consistent with previous studies of rDNA variation in Daphnia [33,37,38]. Although rates of rDNA recombination have not been estimated in D. pulex or D. pulicaria, a rate of 0.02 to 0.06 events per generation was obtained in the congeneric species, $D$. obtusa [33], which is at the high end of rates estimated for rDNA in other organisms $\left(10^{-2}\right.$ to $10^{-5}$ [33]).

It is generally assumed that rDNA loci primarily contain canonical units consisting of one copy of each rRNA gene plus spacers (Figure 1), but this has rarely been tested. Based on this assumption, we expected our estimates of $18 \mathrm{~S}$ and $28 \mathrm{~S}$ number to be very similar within isolates, and they often are (Additional files 2 and 4). However, there are also a substantial number of isolates in which one gene (usually $28 \mathrm{~S}$ ) significantly outnumbers the other (Additional files 2 and 4) suggesting that experimental variation is not likely to account for all these differences. This conclusion is supported by the discovery of unusual rDNA configurations in humans. For example, Caburet et al. [39] used fluorescent in situ hybridization (FISH) and Southern blotting to show that up to one-third of the rDNA units in human fibroblast cell lines consist of head-to-head and tail-to-tail palindromic rearrangements of $18 \mathrm{~S}$ and 28S. Moreover, these noncanonical units sometimes occur in clusters, which is consistent with their amplification by unequal sister chromatid exchange between rDNA units. In addition, Zafiropoulosa et al. [40] used qPCR to measure the number of $18 \mathrm{~S}, 5.8 \mathrm{~S}$ and $28 \mathrm{~S}$ in adipose tissue samples from humans and found significant differences between genes within individuals. They concluded that these differences were a consequence of unequal recombination events initiating between the $18 \mathrm{~S}$ and $5.8 \mathrm{~S}$ and, subsequently, eliminating part of an rDNA unit. These studies suggest that our observation of large differences in $18 \mathrm{~S}$ and $28 \mathrm{~S}$ number in some Daphnia isolates may not be an artefact of qPCR analysis, and thus warrants further study.

\section{Variation in Pokey number}

On average, we found only 2 to 3 rPokey elements per haploid genome (maximum of 12 with three exceptions 
discussed below, Table 1). We also found isolates in which rPokey is completely absent, which is also the case in previous studies [21,41]. In contrast, gPokey is present in all isolates that have been analyzed in this and previous studies [21,23,41]. Indeed, over $75 \%$ of Pokey elements are located outside of $28 \mathrm{~S}$ genes in most isolates. Thus, even if all the genes containing Pokey insertions were eliminated from rDNA, active gPokey elements could eventually "recolonize" it. However, despite the higher number of gPokey, they are also fairly constrained; there are usually less than 10 copies per haploid genome. These results are similar to those obtained by Valizadeh and Crease [21] using TE Display. They estimated Pokey number per diploid genome to be from 1 to 12 in 83 isolates of D. pulex from the Midwest US and Ontario. However, these are likely to be underestimates because Valizadeh and Crease [21] used an annealing temperature of $55^{\circ} \mathrm{C}$. Vergilino [18] analyzed Daphnia isolates using TE Display, but used an annealing temperature of $50^{\circ} \mathrm{C}$. We analyzed a few isolates from his study using an annealing temperature of $55^{\circ} \mathrm{C}$, and obtained about half as many fragments as he did (data not shown). If the gPokey estimates from Valizadeh and Crease [21] were doubled, they would be even closer to the ones we obtained with qPCR (Table 1).

The low number of both rPokey and gPokey in most isolates is consistent with the hypothesis that Pokey activity is generally low. Recombination among rDNA units further increases the rate at which rPokey is eliminated, and can eventually eliminate it altogether in some isolates. However, if Pokey has been completely inactive for a substantial period of time in either species, we would not expect to find any rPokey, although gPokey could persist and even go to fixation by drift in some sites if it did not have deleterious effects on gene structure or function. Nevertheless, we identified three isolates with unusually high numbers of both $\mathrm{rPokey}$ and gPokey, and indeed, these isolates are responsible for the positive correlation between them in D. pulicaria (Figure $4 \mathrm{~b})$. It is unlikely that the high rPokey number in these isolates is a consequence of large changes in the size of the rDNA locus as the two isolates with 40 or more rPokey have less than $15028 \mathrm{~S}$ genes per haploid genome (Additional file 2). Moreover, such changes in rDNA would have little or no impact on gPokey number. Thus, the most likely explanation for the high Pokey number in these isolates is a recent increase in transposition activity.

There are several mechanisms that could explain the putative increase in Pokey activity in these isolates. For example, it has been shown that some TEs are activated during a response to stress ([42] and references within). Alternatively, mutations could have occurred in Pokey that increase its ability to transpose or allow it to avoid silencing by the host, or in the host that decrease its ability to silence Pokey $[3,43]$. A similar scenario was suggested by Eickbush and colleagues [12,44], who showed that levels of R2 transcription can vary up to 100 -fold in natural populations of Drosophila. They explained this by suggesting that $\mathrm{R} 1$ and $\mathrm{R} 2$ serve as foci for the formation of heterochromatin, which deactivates rDNA units. Because active rDNA units in Drosophila tend to occur in contiguous blocks $[45,46]$, individuals are best able to silence these elements when they are clustered with one another in the rDNA array. Conversely, if they have been interspersed with uninserted, functional $28 \mathrm{~S}$ genes by recombination, they are less likely to be silenced and thus show higher rates of transposition.

We do not know how Pokey elements are distributed in the rDNA of Daphnia, but Glass et al. [47] suggested that they are likely to be clustered based on patterns of sequence variation among $28 \mathrm{~S}$ with and without Pokey insertions. In contrast, FISH analysis of one isolate with an IGS and a Pokey probe suggests that its Pokey elements are dispersed throughout the rDNA array [34]. Thus, it is not clear if the relationship between element activity and distribution suggested for R-elements in Drosophila will also be the case for rPokey in Daphnia. However, this could be tested by analyzing the same individuals (or their clonally produced offspring) using both FISH and qPCR.

\section{Variation in 285 gene insertion number}

Based on analysis of $D$. pulex rDNA cloned into a phage vector, Sullender [16] estimated that rPokey elements occupy approximately $10 \%$ of the $28 \mathrm{~S}$ genes. Based on our qPCR analyses, this is a substantial overestimate. However, even though rPokey generally occupies about $1 \%$ of the $28 \mathrm{~S}$ in an isolate, rInserts occupy 5 to $7 \%$ of these genes, on average. Furthermore, unlike rPokey, rInsert number is significantly correlated with t28S number (Table 2, Figure 5), suggesting that rInserts are amplified by the same mechanism that amplifies u28S, and/or their transposition rate increases as $\mathrm{u} 28 \mathrm{~S}$ number increases. This same pattern was observed by Averbeck and Eickbush [32] for R1 in the replicate lines of $D$. melanogaster, although the slope of the line generated by plotting the total number of rRNA genes relative to the number of $\mathrm{R} 1$ insertions was 0.27 , which is two to three times higher than our result of 0.10 for D. pulicaria and 0.15 for D. pulex (Table 2). Averbeck and Eickbush [32] argued that R2 insertions, whose number remained low (as does rPokey in Daphnia), were excluded from recombination events among rDNA units, while R1 elements were underrepresented but still included, which allowed them to increase as the size of 
the rDNA locus increased (as do rInserts in Daphnia). Even so, R1-inserted 28S did not increase at the same rate as u28S, suggesting that their amplification was somehow constrained. The simulation study of Zhang et al. [8] provides an explanation for this behavior. When simulations included elimination of chromosomes with low numbers of $\mathrm{u} 28 \mathrm{~S}$ by natural selection, recombination among rDNA units tended to increase u28S faster than inserted (i) $28 \mathrm{~S}$.

Glass et al. [47] sequenced approximately $850 \mathrm{nt}$ of $28 \mathrm{~S}$ downstream of the Pokey insertion site in 20 isolates of $D$. pulex and found that variation was higher among genes with Pokey than those without. This is consistent with relaxed selection on these presumably non-functional 28S, which allows them to accumulate nt substitutions and short indels that would normally be deleterious. It is also consistent with the hypothesis that the presence of Pokey inhibits recombination between i28S and u28S. This inhibition may also explain why there is no correlation between rPokey and t28S (Table 2 , Figure 5), and why selection is not as efficient at removing $28 \mathrm{~S}$ genes with Pokey insertions, which are much more deleterious than a single nt substitution or short indel.

It is possible that some of the rInserts we detected in Daphnia 28S are R1 and/or R2, but this seems unlikely. First, the insertion site for R1 is not located between the qPCR primers that span the Pokey TTAA insertion site. Second, we have not been able to amplify R2 (whose insertion site is 4 nt downstream of the TTAA) from some of the $D$. pulex and D. pulicaria isolates analyzed in this study using degenerate primers that have been used successfully in a wide range of arthropod species [48]. A more likely possibility is that the rInserts we detected are degraded or divergent Pokey elements, and indeed, a second Pokey lineage has recently been identified in the $D$. pulex genome sequence [15]. Based on its similarity to a divergent lineage that was previously identified in D. obtusa [17], this lineage has been designated PokeyB. In addition, two types of miniature inverted-repeat transposable elements (MITEs) were also identified in the genome sequence. These MITEs are approximately $750 \mathrm{nt}$ in length, and one of them (mPok1) has TIRs similar to the original Pokey lineage, which we now designate as PokeyA, while the other (mPok2) has TIRs similar to PokeyB [15].

Sequence alignment of the four groups of elements suggests that our qPCR primers will not amplify Pokey $\mathrm{B}$ or $\mathrm{mPok} 2$, but they should bind to mPok1. Thus, it is possible that some of the rInserts are members of these two groups. This is supported by the fact that we have been able to amplify PokeyB and mPok2 from the $28 \mathrm{~S}$ of some of our D. pulex and D. pulicaria isolates using a forward primer that is specific to them and a reverse primer in the $28 \mathrm{~S}$ (data not shown). So far, we have not been able to amplify mPok 1 from $28 \mathrm{~S}$, although it is usually present elsewhere in the genome.

Another possibility is that rInserts are partial Pokey elements generated by recombination events that deleted part of the element and possibly part of the rDNA unit in which it resides. This would explain why numbers of PokeyA and $18 \mathrm{~S}$ are similar for both Log50 isolates, but Log50-11 has approximately 240 more copies of $28 \mathrm{~S}$ than $18 \mathrm{~S}$. These additional $28 \mathrm{~S}$ could have inserts (Table 3), or they may be recombinants that did not amplify with the primers spanning the Pokey insertion site. The fact that Pokey contains sequences derived from the ribosomal IGS $[14,15]$ supports the idea that aberrant rDNA units with (partial) Pokey insertions could have been created by recombination between $\mathrm{rPo}$ key and rDNA. If this is the case, we expect these aberrant rDNA units to be deleterious and thus eliminated by natural selection before they expand to such high number. On the other hand, they could persist within populations for a considerable period of time if (1) they are clustered, which could occur if they were amplified by unequal sister chromatid exchange between rDNA units that are offset by one or a few copies, or (2) if they are not transcribed, either because their promoters were deleted by recombination (as suggested in [39] for the human fibroblasts), or they ended up in a region of the rDNA array that is particularly "attractive" to the heterochromatinization machinery (as suggested in [44] for R1 and R2).

Whatever the nature of rInserts in Daphnia 28S genes, it is clear that their dynamics are somewhat different from those of PokeyA. The latter behave like R2 in the replicate D. melanogaster lines [32], while the rInserts behave more like R1. Averbeck and Eickbush [32] attributed the differences between these two elements to two factors; differences in rates of participation in recombination events and differences in transposition rate, which were estimated to be much higher for R1 than R2. If Daphnia rInserts are indeed PokeyB or mPok2, we would expect them to have a higher transposition rate than PokeyA. We are in the process of testing this prediction using yeast excision assays, which have shown that the PokeyA transposase is active [15]. If PokeyB does have a higher excision rate, we would also expect it to be more numerous than PokeyA in other genomic locations as well as rDNA. We also predict that $\mathrm{mPok} 2$ will be more numerous in $28 \mathrm{~S}$ than are full length PokeyA or PokeyB for two reasons. First, MITEs are often found to occur in higher number than the fulllength elements whose transposase they use to move [49]. Second, the much shorter length of mPok2 may reduce or eliminate the bias against recombination between i28S and u28S. This could contribute to the 
significant correlation between $\mathrm{t} 28 \mathrm{~S}$ and rInserts (Table 2, Figure 5). In order to test these predictions, we are now in the process of developing qPCR primers to quantify each of the Pokey and MITE groups separately, both in and outside of $28 \mathrm{~S}$ genes.

\section{Impact of life history variation on rDNA and Pokey dynamics}

Previous studies $[21,23,37,41,47]$ have shown that loss of sexual reproduction in obligately parthenogenetic lineages of $D$. pulex does impact both rDNA and gPokey dynamics. In this study, we compared two Daphnia species whose life histories differ in several ways, including timing and frequency of sexual reproduction, brood size, juvenile growth rate and life span [26]. Overall, patterns of rDNA and Pokey number variation are virtually indistinguishable in the two species (Table 1, Figures 4 and 5). Thus, it seems that their life history differences have little, if any, impact on Pokey dynamics, most likely because "a little bit of sex is nearly as good as a lot" [50]. The only exception is the three D. pulicaria isolates with unusually high rPokey and gPokey loads, which we suggest is a consequence of recent transposition activity. Further analysis of isolates from these populations would likely provide additional insights into Pokey dynamics.

\section{Conclusions}

It is clear that rDNA is very dynamic and much more complicated than once thought. In this study, we observed substantial variation in rDNA copy number among Daphnia isolates, which is consistent with previous studies suggesting that sister chromatid exchange is more frequent than interchromosomal exchange in Daphnia rDNA. Moreover, we sometimes found substantial differences in $18 \mathrm{~S}$ and $28 \mathrm{~S}$ number within isolates, which is not likely to be explained entirely by experimental variation and thus warrants further study. In general, there are less than 20 Pokey elements per haploid genome and most are gPokey. This suggests that transposition rates are generally very low and that recombination, in combination with natural selection, eliminates rPokey faster than gPokey. Even so, three isolates of $D$. pulicaria have unusually high numbers of both rPokey and gPokey, which we suggest is due to a recent increase in transposition activity. We also detected other rDNA insertions (rInserts) that could be degraded Pokey elements, R- elements or members of the divergent PokeyB lineage that was recently detected in the $D$. pulex genome sequence. Although rPokey number is not correlated with $\mathrm{t} 28 \mathrm{~S}$ number, rInsert number is, suggesting that they are amplified by the same mechanisms as rDNA units while rPokey is not. Overall, we observed no impact of life history differences between $D$. pulex and D. pulicaria on the dynamics of either rPokey or gPokey.

\section{Methods}

\section{Daphnia samples and DNA extractions}

A total of 69 Daphnia isolates collected from 16 ponds (D. pulex) and 6 lakes (D. pulicaria) were included in this study (Figure 2, Additional file 1). Ponds were sampled by skimming a dip net just under the surface of the water. Lakes were sampled by towing a net behind a small boat. Clonally-propagated lines were established from single females and grown in $200 \mathrm{~mL}$ of dechlorinated tap water at room temperature. Cultures were fed either live Scenedesmus or frozen Nannochloropsis algae (Landlocked Mariculture LLC, Toronto, Ontario, Canada). Genomic DNA was extracted from multiple individuals from each line using the phenol:chloroform method [51] or the AquaGenomics extraction kit with the manufacturer's tissue protocol (MultiTarget Pharmaceuticals LLC, Salt Lake City, Utah, USA). DNA concentrations were estimated using a NanoDrop ${ }^{\circledR}$ ND-8000 spectrophotometer (Wilmington, Delaware, USA) and ranged from 1 to $1,500 \mathrm{ng} / \mu \mathrm{L}$.

Lab isolates were identified as $D$. pulex or $D$. pulicaria by PCR-amplifying and sequencing an approximately $700 \mathrm{nt}$ fragment of the mitochondrial NADH dehydrogenase 5 (ND5) gene [52]. The breeding system (cyclic or obligate parthenogenesis) of most lab-reared isolates was determined by examination of diapausing egg cases (ephippia) produced in the absence of males. While cyclical parthenogens often release empty ephippial cases unless the eggs have been fertilized, obligate parthenogens deposit eggs into ephippia even in the absence of males [53].

\section{qPCR}

We used the $\Delta C_{\mathrm{T}}$ quantitative (q)PCR method to estimate haploid copy number by comparing the rate of amplification of a multicopy gene to that of two singlecopy genes as in McTaggart et al. [33]. The copy number of 18S, t28S, u28S, rPokey, total Pokey, Tif and Gtp were estimated using seven pairs of primers (Figure 1, Additional file 5). We generated standard curves (Additional file 6) to validate each primer pair and determine its percent amplification efficiency (PAE).

qPCR reactions were $20 \mu \mathrm{L}$ in volume, with $1 \mathrm{X}$ Power SYBR $^{\circledR}$ Green PCR Master Mix (Applied Biosystems, Foster City, California, USA), 0.25 pmol of each primer, and approximately $10 \mathrm{ng}$ of template. Reactions were run on the StepOnePlus ${ }^{\mathrm{TM}}$ Real-Time PCR System (Applied Biosystems). The PCR program was as follows: $95^{\circ} \mathrm{C}$ for 10 minutes, 40 cycles of $95^{\circ} \mathrm{C}$ for $15 \mathrm{sec}$ and $60^{\circ} \mathrm{C}$ for 1 minute. After the 40 cycles were complete, a dissociation curve was created during one additional 
cycle by increasing the temperature from $60^{\circ} \mathrm{C}$ to $95^{\circ} \mathrm{C}$ in increments of $0.3^{\circ} \mathrm{C}$. All reactions were run in triplicate.

The baseline was set automatically by the StepOne v2.0 software (Applied Biosystems). The threshold was set based on amplicon size as larger amplicons bind more SYBR Green and thus produce more fluorescence. This has little effect when gene number is low, but the effect is substantial at high gene numbers. Thus, we set the threshold to 0.2 for the smallest amplicon of $50 \mathrm{bp}$, and calculated the threshold for larger amplicons as 0.2 $\times 2^{\wedge}(1-(50 /$ length in $\mathrm{bp}))$. If the standard deviation of the triplicate mean $C_{T}$ value was larger than 0.2 , we excluded the most extreme replicate. However, if there was no clear outlier, we used all three $C_{T}$ values in the analysis.

We calculated gene number according to [33] with the PAE correction of [54] as $2^{-\Delta C T}$ where $\Delta C_{T}$ is $\left(\left(C_{T} \times\right.\right.$ $\mathrm{PAE}$ multicopy gene $\left.)-\left(\mathrm{C}_{\mathrm{T}} \times \mathrm{PAE}_{\text {single-copy gene }}\right)\right)$. We used all combinations of $\mathrm{C}_{\mathrm{T}}$ values from multi-copy and single-copy gene triplicates to generate a total of 18 estimates $((3 \times 3)+(3 \times 3))$ when all values were included (Additional file 7). These 18 estimates were averaged to give the mean and standard deviation of haploid copy number for each multi-copy gene in each isolate. The haploid numbers were rounded up or down to the nearest 0.5. We used Microsoft Excel (Richmond, Washington, USA) to do correlation and regression analyses, and $\mathrm{t}$-tests. We used the sequential Bonferroni technique of Rice [55] to adjust the significance level (0.05) for t-tests comparing $18 \mathrm{~S}$ and $28 \mathrm{~S}$ number within isolates.

\section{Additional material}

Additional file 1: Population location and sample size. This PDF file provides sample size, latitude and longitude for the 22 Daphnia populations sampled.

Additional file 2: Haploid rRNA gene and Pokey number. This PDF file provides estimates (and standard deviation) of rRNA gene and Pokey number for all Daphnia isolates in this study. Daphnia pulex were collected from ponds $(\mathrm{P})$ and D. pulicaria from lakes $(\mathrm{L})$.

Additional file 3: Tif and Gtp cloning experiment. This PDF file describes the cloning and sequencing of Tif and Gtp genes from four Daphnia isolates with a range of Tif:Gtp ratios. The purpose of this work was to determine if isolates with very low or very high Tif:Gtp ratios possess three alleles at one of the loci.

Additional file 4: Histograms of haploid rRNA gene and insertion number. This is a PDF file. (a) Haploid 185 and 285 gene number in each Daphnia isolate. Vertical lines are standard errors. Differences that are not significant after sequential Bonferroni correction are indicated by "ns". (b) Haploid number of rPokey and $28 \mathrm{~S}$ with and without inserts in each Daphnia isolate. u28S are uninserted 285 genes, rPokey are inserted in $28 \mathrm{~S}$, rlnserts are inserts other than rPokey in $28 \mathrm{~S}$. The number of r/nserts was calculated as (total 28S-uninserted 28S-rPokey).

Additional file 5: qPCR primers. This PDF file provides sequences for GPCR primers, as well as the threshold value and the percent amplification efficiency (PAE) for each primer pair.
Additional file 6: Standard curve analysis for qPCR primers. This PDF file provides details of the standard curve experiments used to validate the $\mathrm{qPCR}$ primers, including a description of the plasmid clones used as templates.

Additional file 7: $C_{T}$ values for all $q P C R$ reactions. This Excel spreadsheet provides $C_{T}$ values for all Daphnia isolates and $\mathrm{qPCR}$ amplicons. Replicates that were omitted from the analyses are highlighted in grey. A template for calculating gene number using the $\Delta C_{T}$ method is also provided, with sample calculations for isolate P6.7.

\section{Abbreviations}

18S: $18 \mathrm{~S}$ rRNA gene; 28S: $28 \mathrm{~S}$ rRNA gene; bp: base pair; $\mathrm{C}_{\mathrm{T}}$ : threshold cycle; ETS: external transcribed spacer; FISH: fluorescent in-situ hybridization; gPokey: Pokey elements found outside of rDNA; i28S-inserted $28 \mathrm{~S}$ rRNA gene; IGS: intergenic spacer; ITS: internal transcribed spacer; kb: kilobase pair; LTR: long-terminal repeat; MITE: miniature inverted-repeat transposable element; nt: nucleotide; PAE: percent amplification efficiency; qPCR: quantitative PCR; rDNA: ribosomal DNA; rInserts: rDNA insertions other than rPokey; rPokey: Pokey elements found in rDNA; rRNA: ribosomal RNA; t28S: total $28 \mathrm{~S}$ rRNA genes; TE: transposable element; TG ratio: Tif to Gtp ratio; TIR: terminal inverted repeat; U28S: uninserted $28 \mathrm{~S}$ rRNA gene.

\section{Acknowledgements}

Funding for this work was provided by an NSERC Discovery Grant to TJC. SHCE was supported by an Ontario Graduate Studies Science and Technology Scholarship. We thank Melania Cristescu for providing some of the Daphnia samples, and the Genomics Facility at the University of Guelph for assistance with the qPCR and sequencing of plasmid clones. Comments from Tyler Elliott and three anonymous reviewers improved earlier versions of the manuscript.

\section{Authors' contributions}

SHCE collected and analyzed the data. TJC conceived the study and supervised the work. Both authors wrote the manuscript and approved the final version.

\section{Competing interests}

The authors declare that they have no competing interests.

Received: 26 August 2011 Accepted: 5 March 2012

Published: 5 March 2012

\section{References}

1. Hua-Van A, Le Rouzic A, Boutin TS, Filée J, Capy P: The struggle for life of the genome's selfish architects. Biol Direct 2011, 6:19.

2. Feschotte C, Pritham EJ: DNA transposons and the evolution of eukaryotic genomes. Ann Rev Genet 2007, 41:331-368.

3. Nuzhdin SV: Sure facts, speculations, and open questions about the evolution of transposable element copy number. Genetica 1999, 107:129-37.

4. Slotkin RK, Martienssen R: Transposable elements and the epigenetic regulation of the genome. Nat Rev Genet 2007, 8:272-285.

5. Fedoroff N: Control of mobile DNA. In Mobile DNA II. Edited by: Craig NL, Craige R, Gellert M, Lambowitz AM. Washington DC: ASM Press; 2002:997-1007

6. Labrador M, Corces VG: Interactions between transposable elements and the host genome. In Mobile DNA II. Edited by: Craig NL, Craige R, Gellert M, Lambowitz AM. Washington DC: ASM Press; 2002:1008-1023.

7. Eickbush $\mathrm{TH}$, Eickbush DG: Finely orchestrated movements: evolution of the ribosomal RNA genes. Genetics 2007, 175:477-485.

8. Zhang X, Eickbush MT, Eickbush TH: Role of recombination in the longterm retention of transposable elements in rRNA gene loci. Genetics 2008, 180:1617-1627

9. Kojima KK, Fujiwara H: Evolution of target specificity in R1 clade non-LTR retrotransposons. Mol Biol Evol 2003, 20:351-361.

10. Kojima KK, Kuma K, Toh H, Fujiwara H: Identification of rDNA-specific nonLTR retrotransposons in Cnidaria. Mol Biol Evol 2006, 23:1984-1993. 
11. Eickbush TH: R2 and related site-specific non-long terminal repeat retrotransposons. In Mobile DNA II. Edited by: Craig NL, Craige R, Gellert M, Lambowitz AM. Washington DC: ASM Press; 2002:813-835.

12. Eickbush DG, Ye J, Zhang X, Burke WD, Eickbush TH: Epigenetic regulation of retrotransposons within the nucleolus of Drosophila. Mol Cell Biol 2008, 28:6452-6461.

13. Gladyshev EA, Arkhipova IR: Rotifer-specific R9 retrotransposable elements generate an exceptionally long target site duplication upon insertion. Gene 2009, 448:145-150.

14. Penton EH, Sullender BW, Crease TJ: Pokey, a new DNA transposon in Daphnia (Cladocera: Crustacea). J Mol Evol 2002, 55:664-673.

15. Elliott T: The activity and evolution of the Daphnia DNA transposon Pokey. MSc thesis University of Guelph, Department of Integrative Biology; 2011.

16. Sullender BW: Preliminary characterization and population survey of the Daphnia rDNA transposable element, Pokey. PhD thesis University of Oregon, Department of Biology; 1993.

17. Penton EH, Crease TJ: Evolution of the transposable element Pokey in the ribosomal DNA of species in the subgenus Daphnia (Crustacea: Cladocera). Mol Biol Evol 2004, 21:1727-1739.

18. Vergilino R: Hybridization and polyploidy in the Daphnia pulex species complex and its effects on the evolution of a transposon. PhD thesis University of Quebec at Rimouski, Department of Biology; 2011.

19. Xu H-F, Xia Q-Y, Liu C, Cheng T-C, Zhao P, Duan J, Zha X-F, Liu S-P: Identification and characterization of piggyBac-like elements in the genome of domesticated silkworm, Bombyx mori. Mol Genet Genomics 2006, 276:31-40.

20. Jurka J: Repbase update: a database and an electronic journal of repetitive elements. Trends Genet 2000, 9:418-420.

21. Valizadeh P, Crease TJ: The association between breeding system and transposable element dynamics in Daphnia pulex. J Mol Evol 2008, 66:643-654.

22. Schaack S, Choi E, Lynch M, Pritham EJ: DNA transposons and the role of recombination in mutation accumulation in Daphnia pulex. Genome Biol 2010, 11:R46.

23. Schaack S, Pritham EJ, Wolf A, Lynch M: DNA transposon dynamics in populations of Daphnia pulex with and without sex. Proc Biol Sci 2010, 277:2381-2387.

24. Černý M, Hebert PDN: Genetic diversity and breeding system variation in Daphnia pulicaria from North American lakes. Heredity 1993, 71:497-507.

25. Tessier AJ, Welser J: Cladoceran assemblages, seasonal succession and the importance of a hypolimnetic refuge. Freshwater Biol 1991, 25:85-93.

26. Dudycha $J \mathrm{~L}$, Tessier AJ: Natural genetic variation of life span, reproduction, and juvenile growth in Daphnia. Evolution 1999, 53:1744-1756

27. Dudycha JL: Mortality dynamics of Daphnia in contrasting habitats and their role in ecological divergence. Freshwater Biol 2004, 49:505-514.

28. Hebert PDN, Beaton MJ, Schwartz SS, Stanton DJ: Polyphyletic origins of asexuality in Daphnia pulex. I. Breeding-system variation and levels of clonal diversity. Evolution 1989, 43:1004-1015.

29. Omilian AR, Lynch M: Patterns of intraspecific DNA variation in the Daphnia nuclear genome. Genetics 2009, 182:325-336.

30. Wright SI, Le QH, Schoen DJ, Bureau TE: Population dynamics of an Aclike transposable element in self- and cross-pollinating Arabidopsis. Genetics 2001, 158:1279-1288.

31. Nuzhdin SV, Petrov DA: Transposable elements in clonal lineages: lethal hangover from sex. Biol J Linn Soc Lond 2003, 79:33-41.

32. Averbeck KT, Eickbush TH: Monitoring the mode and tempo of concerted evolution in the Drosophila melanogaster rDNA locus. Genetics 2005, 171:1837-1846.

33. MCTaggart SJ, Dudycha JL, Omilian A, Crease TJ: Rates of recombination in the ribosomal DNA of apomictically propagated Daphnia obtusa lines. Genetics 2007, 175:311-320.

34. Colbourne JK, Pfrender ME, Gilbert D, Thomas WK, Tucker A, Oakley TH, Tokishita S, Aerts A, Arnold GJ, Basu MK, Bauer DJ, Caceres CE, Carmel L, Casola C, Choi J-H, Detter JC, Dong Q, Dusheyko S, Eads BD, Frohlich T, Geiler-Samerotte KA, Gerlach D, Hatcher P, Jogdeo S, Krijgsveld J, Kriventseva EV, Kultz D, Laforsch C, Lindquist E, Lopez J, et al: The ecoresponsive genome of Daphnia pulex. Science 2011, 331:555-561.
35. Weider L, Elser J, Crease T, Mateos M, Cotner J, Markow T: The functional significance of ribosomal(r)DNA variation: Impacts on the evolutionary ecology of organisms. Ann Rev Ecol Evol Syst 2005, 36:219-242.

36. Paredes S, Branco AT, Hartl DL, Maggert KA, Lemos B: Ribosomal DNA deletions modulate genome-wide gene expression: "rDNA-sensitive" genes and natural variation. PLOS Genet 2011, 7:e1001376.

37. Crease TJ, Lynch M: Ribosomal DNA variation in Daphnia pulex reproducing by cyclic and obligate parthenogenesis. Mol Biol Evol 1991, 8:620-640.

38. Crease T: Ribosomal DNA evolution at the population level: nucleotide variation in intergenic spacer subrepeat arrays of Daphnia pulex. Genetics 1995, 141:1327-1337

39. Caburet S, Conti C, Schurra C, Lebofsky R, Edelstein SJ, Bensimon A: Human ribosomal RNA gene arrays display a broad range of palindromic structures. Genome Res 2005, 15:1079-1085.

40. Zafiropoulosa A, Tsentelieroua E, Linardakisb M, Kafatosb A, Spandidos DA: Preferential loss of $5 S$ and $28 \mathrm{~S}$ rDNA genes in human adipose tissue during ageing. Intl J Biochem Cell Biol 2005, 37:409-415.

41. Sullender BW, Crease TJ: The behavior of a Daphnia pulex transposable element in cyclically and obligately parthenogenetic populations. J Mol Evol 2001, 53:63-69.

42. Capy P, Gasperi G, Biémont C, Bazin C: Stress and transposable elements: co-evolution or useful parasites? Heredity 2000, 85:101-106.

43. Deininger PL, Roy-Engel AM: Mobile elements in animal and plant genomes. In Mobile DNA II. Edited by: Craig NL, Craige R, Gellert M, Lambowitz AM. Washington DC: ASM Press; 2002:1074-1092.

44. Zhou J, Eickbush $\mathrm{TH}$ : The pattern of R2 retrotransposon activity in natural populations of Drosophila simulans reflects the dynamic nature of the rDNA locus. PLoS Genet 2009, 5:1000386.

45. Chooi WY: The occurrence of long transcription units among the $X$ and $Y$ ribosomal genes of Drosophila melanogaster: transcription of insertion sequences. Chromosoma 1979, 74:57-74.

46. Jamrich M, Miller OL: The rare transcripts of interrupted rRNA genes in Drosophila melanogaster are processed or degraded during synthesis. EMBO J 1984, 3:1541-1545.

47. Glass S, Mozczynska A, Crease T: The effect of transposon Pokey insertions on sequence variation in the 28S rRNA gene of Daphnia pulex. Genome 2008, 51:988-1000.

48. Burke WD, Malik HS, Jones JP, Eickbush TH: The domain structure and retrotransposition mechanism of R2 elements are conserved throughout arthropods. Mol Biol Evol 1999, 16:502-511.

49. Feschotte $C$, Zhang $X$, Wessler S: Miniature inverted-repeat transposable elements (MITEs) and their relationship with established DNA transposons. In Mobile DNA II. Edited by: Craig NL, Craige R, Gellert M, Lambowitz AM. Washington DC: ASM Press; 2002:1147-1158.

50. Green RF, Noakes DLG: Is a little bit of sex as good as a lot? I Theor Biol 1995, 174:87-96

51. Sambrook J, Fritsch EF, Maniatis T: Molecular Cloning: A Laboratory Manual. 2 edition. Cold Spring Harbor, NY: Cold Spring Harbor Laboratory Press; 1989.

52. Cristescu M, Constantin A, Bock D, Cáceres C, Crease T: Speciation with gene flow and the genetics of habitat transitions in the Daphnia pulex species complex. Mol Ecol 2012, Epub ahead of print.

53. Innes DJ, Schwartz SS, Hebert PDN: Genotypic diversity and variation in mode of reproduction among populations in the Daphnia pulex group. Heredity 1986, 57:345-355.

54. Yuan JS, Wang D, Stewart CN: Statistical methods for efficiency adjusted real-time PCR quantification. Biotechnol J 2008, 3:112-123.

55. Rice WR: Analyzing tables of statistical tests. Evolution 1989, 43:223-225.

\section{doi:10.1186/1759-8753-3-4}

Cite this article as: Eagle and Crease: Copy number variation of ribosomal DNA and Pokey transposons in natural populations of Daphnia. Mobile DNA 2012 3:4. 